\title{
Avaliação psicológica da personalidade de condutores: uma revisão de literatura
}

\author{
Fábio Henrique Vieira de Cristo e Silva - Universidade Federal do Rio Grande do Norte ${ }^{1}$ \\ João Carlos Alchieri - Universidade Federal do Rio Grande do Norte
}

\begin{abstract}
Resumo
Todos os anos milhões de brasileiros são avaliados psicologicamente para conduzir veículos, com base na legislação vigente. $\mathrm{O}$ objetivo deste trabalho foi revisar os estudos empíricos brasileiros sobre avaliação psicológica de características da personalidade utilizados nos processos avaliativos de condutores. Identificaram-se publicações nesta temática no país, os principais temas e métodos utilizados, bem como listaram-se os procedimentos e resultados obtidos. Conclui-se que as pesquisas sobre personalidade de motoristas são escassas e restritas nos seus resultados com base na generalização, sem resultados conclusivos que justifiquem a necessidade de avaliação deste construto psicológico para conduzir veículos.

Palavras-chave: Personalidade de condutores; Avaliação psicológica de motoristas; Motoristas; Revisão de literatura; Psicologia do trânsito.
\end{abstract}

\section{Psychological assessment of the personality of drivers: a review of literature}

\begin{abstract}
Every year millions of Brazilians are psychologically assessed in order to drive vehicles, according to the current legislation. The object of this work was review the Brazilian empirical studies on the psychological assessment of the personality characteristics used in the evaluation processes of drivers. The publications on this subject in the country were identified, as well as their main themes and methods used; and their procedures and results obtained were listed. It is concluded that the researches on the personality of drivers are scarce and restricted in their results, based on generalizations and without offering conclusive results which justify the need to evaluate this psychological construct in order to drive vehicles.

Keywords: Personality of drivers; Psychological evaluation of drivers; Drivers; Review of literature; Traffic psychology.
\end{abstract}

\section{Introdução}

No Brasil, a história da psicologia aplicada ao trânsito remonta à década de 1930, quando se iniciaram as primeiras aplicações de instrumentos psicológicos de orientação e seleção profissional dos futuros profissionais das ferrovias em São Paulo. Nas décadas posteriores, principalmente 1950 e 1960, em razão do avanço da indústria automobilística e do aumento da demanda por segurança, formação e orientação dos condutores, a psicologia do trânsito direcionou suas atividades para o transporte rodoviário, a fim de tentar frear o aumento nos índices de acidentes (Mange, 1956; Trench, 1956). Desse modo, desde 1951 até hoje, a avaliação psicológica (psicotécnico) se inseriu no processo de habilitação, sendo atualmente uma etapa preliminar, obrigatória, eliminatória e complementar para todos os condutores e candidatos a obtenção da habilitação.

Não obstante tais obrigatoriedades, considerados estudos nacionais e internacionais apontam: a) existência de poucas pesquisas que comprovam a validade e fidedignidade da avaliação e dos instrumentos psicológicos aplicados em motoristas (Groeger, 2003); b) ausência de critérios precisos para avaliar o condutor (Alchieri \& Stroeher, 2002, 2003); c) deficiente capacitação profissional dos psicólogos (Rozestraten, 1982; Sbardelini, 1990); d) má qualidade do processo de avaliação psicológica, conforme realizado em alguns departamentos de trânsito e clínicas credenciadas - por exemplo, espaços inapropriados para a aplicação, simplificações e erros no uso dos testes, instrumentos copiados e estabelecimento de critérios menos rigorosos para alguns candidatos (Alves, 1999; Arsênio, 1999; Méa \& Ilha, 2003; Quintela, 1977; Wechsler, 1999); e e) divergências de posicionamentos entre psicólogos peritos examinadores, estudantes de psicologia e usuários, quanto ao grau de importância dado ao processo de avaliação de motoristas (Gouveia e cols., 2002; Lamounier \& Rueda, 2005b).

\footnotetext{
${ }^{1}$ Endereço para correspondência:

Avenida Rui Barbosa, 1.122 - apto 801-B - Lagoa Nova - 59056-300 - Natal-RN

Telefone: (84) 3221-2527

E-mail: fabiodecristo@gmail.com
} 
Essas questões podem ser especialmente delicadas em relação ao construto personalidade. Em alguns países da Europa, por exemplo, a avaliação da personalidade com fins de habilitação de motoristas não é uma obrigação, não somente pela validade insuficiente, senão pelo respeito à individualidade (Rozestraten, 1985). No Brasil, tal avaliação é uma prática bastante difundida e utilizada (Mira, 1984b; Quintela, 1977; Rozestraten, 1990; Trench, 1956), sendo a personalidade considerada causa dos acidentes (por exemplo, Vieira, Amorim \& Carvalho, 1956). Contudo, parece não existir evidências empíricas fortes que sustentem este argumento. Mira (1984a), por exemplo, em revisão sobre a avaliação da personalidade através do Psicodiagnóstico Miocinético (PMK), ainda não havia identificado até meados da década de 1980 nenhum estudo no país que diferenciasse claramente grupos de motoristas pluriacidentados e não-acidentados. Em outro estudo, Duarte (1999) pesquisou dois instrumentos de personalidade para verificação da validade preditiva em relação aos comportamentos de 182 motoristas e não encontrou correlações entre os resultados do Bender projetivo e os comportamentos infratores; já no Inventário Fatorial de Personalidade (IFP), as únicas correlações significativas ocorreram entre o número total de infrações e os fatores assistência e deferência. Em relação aos instrumentos e aos critérios de avaliação, parece também não haver um consenso. Alchieri e Stroeher (2003), por exemplo, observaram a diversidade de instrumentos e critérios utilizados pelos psicólogos, bem como a falta de padronização no processo avaliativo, sendo identificados 14 instrumentos usados para avaliar personalidade em todo o país e um total de 2.056 indicadores para classificar $\mathrm{o}$ indivíduo apto/inapto.

Percebe-se, por essas discussões, que o campo da avaliação psicológica de motoristas é marcado por dificuldades e limitações em sua fundamentação e exercício, não existindo consenso sobre sua validade em relação ao aumento da segurança nos deslocamentos nas vias públicas. É indispensável, portanto, que os psicólogos investiguem e reflitam sistematicamente sobre essa prática avaliativa, uma vez que milhões de brasileiros se submetem todos os anos aos exames psicológicos para obter a permissão para dirigir ou renová-la, que pode estar levando indivíduos aptos a ser considerados inaptos e viceversa (Quintela, 1977). Com isso, os psicólogos podem estar colaborando injustamente com a desigualdade de direitos na circulação dos cidadãos, em suas possibilidades de ir e vir (Rozestraten, 1985). Diante dessas considerações, o objetivo do presente trabalho é contribuir com o aprofundamento das discussões de cunho teóricometodológico, apresentando uma revisão dos estudos empíricos sobre as características da personalidade de condutores no Brasil.

\section{Método}

A fim de atingir o objetivo proposto, buscou-se abarcar a totalidade dos estudos realizados nas últimas cinco décadas, de 1956 a 2006, período este definido do primeiro até os mais recentes trabalhos nessa área, caracterizando-os em relação aos principais temas e métodos utilizados (participantes, instrumentos e delineamentos). A busca pelas publicações ocorreu em bases de dados eletrônicas e em referências bibliográficas de trabalhos de revisão de literatura que identificaram grande parte da produção científica em avaliação psicológica de condutores e em psicologia do trânsito (Alchieri \& Scheffel, 2000; Alchieri \& Stroeher, 2002, 2003; Alves, 1999; Mira, 1984a; Vieira e col., 1956). Essas estratégias foram necessárias à medida que as pesquisas em avaliação psicológica de condutores encontram-se bastante dispersas, inclusive em fontes já fora de circulação (Alchieri \& Scheffel, 2000).

\section{Resultados}

Foram identificados 15 artigos empíricos em periódicos científicos, distribuídos da seguinte forma: um na década de 1950, um na década de 1970, três trabalhos na década de 1990 e dez no período de 2000 a 2006. Ressalta-se que não foi identificado nenhum trabalho de investigação empírica sobre avaliação de aspectos da personalidade em condutores nas décadas de 1960 e 1980. A seguir, são apresentadas resenhas individuais de cada uma das pesquisas, contemplando suas principais características. Para uma visão geral desses conteúdos, ver Tabela 1.

O primeiro trabalho identificado na literatura sobre pesquisa e personalidade foi o de Vieira e cols. (1956), que investigaram o uso do PMK na seleção de motoristas. A amostra foi aleatória com 4.935 motoristas. Os autores apontaram que 33,10\% dos motoristas foram inabilitados no PMK por serem portadores de fortes traços de hiperagressividade e hiperemotividade, aliados a outros distúrbios. Chamaram a atenção para o estudo da personalidade e dos fatores patológicos, salientando o perigo dos emotivos e dos hiperagressivos ao volante.

Boccalandro, Tolentino, Nogueira, Maia e Lima (1971) realizaram estudo com 200 condutores do sexo masculino, com nível de instrução variando da alfabetização ao ensino superior, para construir tabela do PMK para motoristas profissionais de São Paulo. Foram acrescentadas no teste novas medidas, com a finalidade de medir quantitativamente a disritmia. Os autores concluíram que os dados confirmam a hipótese de que as características de personalidade do grupo estudado difeririam da amostra de padronização, proveniente de 
níveis socioeconômicos mais altos do Rio de Janeiro, sendo o grupo de São Paulo, em geral, mais excitado, agressivo, impulsivo e com tônus vital maior do que o grupo de padronização.

Hashimoto e Ribeiro (1991) verificaram se o Bender projetivo seria adequado para avaliar indicadores de personalidade na seleção profissional para o cargo de motorista, verificando a relação do perfil do candidato ao cargo com os indicadores de personalidade apontados no instrumento. Concorreram 77 candidatos a uma vaga de motorista em empresa privada. Foram selecionados no teste 44 indicadores emocionais e 10 físicos, considerados importantes para dirigir. Os autores concluíram que os resultados comprovam as contribuições do Bender projetivo para o processo seletivo de motoristas como um instrumento alternativo (tempo de aplicação reduzido, facilidade de aplicação, custo baixo) e que seria viável realizar apenas a reprodução das figuras geométricas (cópia).

Grisci (1991) investigou a relação entre acidentes de trânsito e as variáveis agressividade, atuação (acting-out) e culpa no teste Gestáltico Visomotor de Bender. Participaram da pesquisa 60 motoristas profissionais de uma empresa, divididos em dois grupos, um com histórico de acidentes de trânsito e outro sem histórico. Os resultados apontaram que o primeiro grupo apresentou um nível significativamente maior de agressividade e atuação em relação ao segundo grupo, apresentando ainda indicadores de agressividade em maior escala, dificuldade no controle dos impulsos agressivos em relação a si e ao meio ambiente, bem como dificuldade em relação a padrões e limites preestabelecidos quanto às leis de trânsito e suas normas de segurança.

Alves e cols. (1998) usaram o PMK abreviado em 114 motoristas profissionais de três níveis de escolaridade, verificando diferenças em relação aos traços de personalidade. Os pesquisadores objetivaram determinar se a escolaridade era uma fator importante nas análises dos resultados do PMK de motoristas e concluíram que não haveria diferenças estatisticamente significativas para nenhuma das 58 variáveis do instrumento.

Bardella, Alves, Esteves, Guedes e Almeida (2001) realizaram um estudo com 211 candidatos à $\mathrm{CNH}$ (Carteira Nacional de Habilitação) para: desenvolver normas para motoristas da cidade do Recife-PE com o PMK (formato abreviado), comparar os resultados entre homens e mulheres e comparar os resultados do grupo de homens com o das tabelas originais do manual do PMK. Comparando homens e mulheres, foram encontradas diferenças significativas apenas em 8 medidas (total de 64), justificando a necessidade de elaboração de tabelas diferenciadas por sexo. Quanto à comparação do grupo masculino com a tabela do manual (tabela A - motoristas

Psico-USF, v. 12, n. 2, p. 189-196, jul./der. 2007 predominantemente brancos), 28 medidas foram diferentes, o que, segundo os autores, confirmaria a necessidade de elaboração de tabelas para as diversas regiões do país.

Costa (2001) desenvolveu estudo com 227 candidatos à $\mathrm{CNH}$ a fim de elaborar tabela de normatização do PMK (formato abreviado) para a cidade de Belo Horizonte-MG, verificando diferenças entre homens e mulheres. Das 66 comparações realizadas, apenas 4 apresentaram diferenças significativas. De acordo com o autor, não haveria justificativa para a adoção de tabelas de tetrons e faixas de aprovação distintas para os dois grupos.

Frasson e Souza (2002) desenvolveram estudo sobre a personalidade de 10 motoristas que tiveram o direito de dirigir suspenso, utilizando o Rorschach e a Escala Diagnóstica Adaptativa Operacionalizada (EDAO). Os instrumentos sinalizaram dificuldades afetivorelacionais (distanciamento nas relações interpessoais e desvalorização do outro) e sociocultural (menor consciência da responsabilidade sobre os atos e carência de limites; predomínio de relações insatisfatórias com os pais, possibilitando o desencadeamento de dificuldades com a autoridade; e interferência da ansiedade, produzindo distúrbio do sono e da alimentação).

Esteves, Bardella, Almeida, Bardella e Alves (2004) compararam as médias dos resultados do PMK (formato abreviado) obtidas em Belo Horizonte com as de Recife, São Paulo e manual do PMK (tabela A - pessoas predominantemente brancas). Participam 356 candidatos à $\mathrm{CNH}$ do sexo masculino. Os resultados mostraram que: entre Belo Horizonte e Recife, foram encontradas 12 diferenças significativas; entre Belo Horizonte e São Paulo, 18 diferenças (em 5 das 6 características avaliadas); entre Belo Horizonte e a tabela A do manual, 23 diferenças significativas (em todas as características avaliadas). Os autores concluíram que seria necessário construir tabelas com normas para diferentes regiões do país.

Lamounier e Rueda (2005a) realizaram investigação com 110 candidatos à $\mathrm{CNH}$ para identificar, através do PMK, possíveis diferenças de variáveis de personalidade entre aptos e inaptos para dirigir e comparar as avaliações dadas pelos dois peritos. A variável agressividade apresentou diferenças significativas, os inaptos com auto-agressividade mais elevada e sem um controle adequado, sendo também considerados mais submissos e passivos, com dificuldades de tomar decisões. Quanto aos pareceres, houve discordância em apenas dois casos, o que, segundo os autores, refutaria afirmações de que a avaliação psicológica de motoristas é feita sem parâmetros preestabelecidos.

Ávila e Benczik (2005) realizaram estudo dos exames psicológicos de um motorista infrator com as 
seguintes características: 28 anos, solteiro, curso superior incompleto, motorista profissional há 10 anos, tendo se envolvido em três acidentes. Utilizando o PMK em formato reduzido, evidenciaram-se: alterações no desvio secundário sagital (DSS); traçado disrítimico (irregular) em todas as folhas e resultado inadequado no DPS; traços disrítmicos, no sentido específico das chamadas lesões cerebrais mínimas. O motorista foi diagnosticado como portador de TDAH (transtorno de déficit de atenção e hiperatividade) do tipo combinado.

Ral, Sanzano e Martinez (2005) realizaram pesquisa com 505 alunos universitários para construir normatização dos lineogramas do PMK-RD (versão digitalizada do PMK) para Barcelona, na Espanha, e compará-la com as de Recife-PE. Os resultados sugeriram que, em Barcelona, não haveria diferenças entre homens e mulheres, e que das 11 diferenças significativas, 7 corresponderiam à mão dominante, o que foi interpretado como uma maior contribuição das diferenças culturais. Os autores concluíram que as diferenças significativas encontradas não foram devidas à utilização de uma forma digitalizada ou analógica do PMK.

Ral, Viadé e Muiños (2006) mostraram dois estudos: um apresentando resultados de comparações dos lineogramas do PMK-RD entre duas amostras de Barcelona e outro comparando resultados dos lineogramas do PMK de Minas Gerais com os do PMK-RD de Barcelona. No estudo 1, as amostras foram compostas por 505 participantes (Ral e cols., 2005) e 248 participantes. No estudo 2, a amostra foi composta por 317 participantes preparando a habilitação (Barcelona) e requerentes à $\mathrm{CNH}$ (Minas Gerais). Foram verificadas diferenças apenas na mão dominante dos desvios secundários entre as duas amostras de Barcelona. Entre Barcelona e Minas Gerais, a maioria das diferenças aconteceram nos desvios secundários, sendo elas atribuídas mais às características de cada amostra do que aos diferentes sistemas de avaliação utilizados (PMK/PMK-RD).

Lamounier e Rueda (2006) verificaram, através do PMK, diferenças de gênero, escolaridade e idade em 126 candidatos à $\mathrm{CNH}$. Os autores concluem que os resultados devem ser avaliados com cautela, pois das 60 medidas avaliadas, apenas cinco mostraram diferenças significativas, uma em relação ao gênero e duas em razão da idade e escolaridade, respectivamente.

Finalmente, Lamounier e Villemor-Amaral (2006) compararam os indicadores de controle de impulsos, afetividade, agressividade e adequação às normas no Método de Rorschach, com o objetivo de buscar evidências de validade desse instrumento. Participaram 42 condutores, divididos em dois grupos (21 infratores de trânsito e 21 não-infratores). Os resultados mostraram diferenças estatisticamente significativas nos seguintes indicadores: Nota AdjD, $\mathrm{Xu} \%, \mathrm{AG}$ y $\mathrm{FC}: \mathrm{CF}+\mathrm{C}$. As autoras concluíram que os resultados sugeririam evidência de validade para o Rorschach no contexto do trânsito, sendo o teste sensível para identificar características psicológicas de indivíduos que poderiam envolver-se em acidentes (ver Tabela1).

\section{Discussão}

Levando-se em consideração a literatura pesquisada, observa-se significativa escassez de publicações, uma vez que apenas quinze artigos são constantemente referenciados na avaliação psicológica de aspectos da personalidade de condutores no Brasil, em cinqüenta anos desta prática. É oportuno destacar ainda que, por duas décadas, não foi encontrado qualquer estudo empírico que embasasse a manutenção da necessidade de avaliação do referido construto. Não obstante, escassos resultados fornecem justificativas para a avaliação da personalidade de condutores e amparam o processo até hoje.

Sobre as temáticas abordadas pelos pesquisado-res, observam-se: cinco estudos sobre validade e padronização de instrumentos (Hashimoto \& Ribeiro, 1991; Lamounier \& Rueda, 2005a; Lamounier \& Villemor-Amaral, 2006; Ral e cols., 2005, 2006), quatro investigando diferenças de personalidade quanto ao gênero, idade e/ou nível educacional (Alves e cols., 1998; Bardella e cols., 2001; Costa, 2001; Lamounier \& Rueda, 2006), quatro estudos com finalidade de construção de tabelas padronizadas (Bardella e cols., 2001; Boccalandro e cols., 1971; Costa, 2001; Ral e cols., 2005), três estudos investigando a relação entre acidentes, infrações e variáveis de personalidade (Ávila \& Benczik, 2005, Frasson \& Souza, 2002; Grisci, 1991), dois efetuando comparação de medidas das tabelas padronizadas em diferentes estados (Boccalandro e cols., 1971; Esteves e cols., 2004) e um realizando investigação das características dos sujeitos pesquisados nos instrumentos de aptidão e personalidade (Vieira e cols., 1956). Pela análise das temáticas abordadas nas investigações, evidenciam-se os poucos estudos sobre validade e padronização de instrumentos, aspecto este já apontado por diversos autores (Alchieri \& Stroeher, 2002, 2003; Duarte, 1999; Groeger, 2003; Mira, 1984a; Sbardelini, 1990). Isso torna extremamente duvidosa a eficácia dos exames psicológicos no processo de habilitação; mesmo assim, o Brasil é o único país que obriga as pessoas a se submeterem a eles. Outro dado que merece destaque, é que apenas três estudos investigaram a relação entre acidentes, infrações e variáveis de personalidade, aspecto fundamental para atribuição de causalidade à personalidade; desse modo, sem muitas evidências, tal causalidade não passa de conjectura, tal qual formulada por Rozestraten (1990), sem comprovação empírica consistente.

Psico-USF, v. 12, n. 2, p. 189-196, jul./der. 2007 
Tabela 1 - Estudos brasileiros em avaliação psicológica da personalidade de condutores

\begin{tabular}{|c|c|c|c|c|c|}
\hline \multirow{2}{*}{ Autores } & \multirow{2}{*}{ Ano } & \multirow{2}{*}{ Temas de investigação } & \multicolumn{3}{|r|}{ Métodos } \\
\hline & & & Particip. & Instrum. & Delineamentos \\
\hline $\begin{array}{l}\text { Vieira e } \\
\text { cols. }\end{array}$ & 1956 & $\begin{array}{l}\text { Características dos sujeitos pesquisados em } \\
\text { instrumentos de personalidade }\end{array}$ & 4.935 & PMK & $\begin{array}{l}\text { Divisão dos participantes em } \\
\text { dois ou mais grupos } \\
\text { comparando-os }\end{array}$ \\
\hline $\begin{array}{l}\text { Boccalandro } \\
\text { e cols. }\end{array}$ & 1971 & $\begin{array}{l}\text { Construção de tabelas padronizadas } \\
\text { Comparação de tabelas padronizadas entre } \\
\text { diferentes estados }\end{array}$ & 200 & $P M K$ & $\begin{array}{l}\text { Comparação entre amostras de } \\
\text { diferentes contextos brasileiros }\end{array}$ \\
\hline $\begin{array}{l}\text { Hashimoto } \\
\text { e Ribeiro }\end{array}$ & 1991 & Estudos sobre validade e padronização & 77 & $\begin{array}{l}\text { Bender } \\
\text { projetivo }\end{array}$ & $\begin{array}{l}\text { Um grupo de sujeitos e verificação do } \\
\text { resultado no(s) instrumento(s) }\end{array}$ \\
\hline Grisci & 1991 & $\begin{array}{l}\text { Relação entre acidentes, infrações e } \\
\text { variáveis de personalidade }\end{array}$ & 60 & $\begin{array}{l}\text { Bender } \\
\text { projetivo }\end{array}$ & $\begin{array}{l}\text { Divisão dos participantes em } \\
\text { dois ou mais grupos } \\
\text { comparando-os }\end{array}$ \\
\hline $\begin{array}{l}\text { Alves e } \\
\text { cols. }\end{array}$ & 1998 & $\begin{array}{l}\text { Relação entre traços de personalidade e } \\
\text { gênero, idade e/ ou nivel educacional }\end{array}$ & 114 & PMK & $\begin{array}{l}\text { Divisão dos participantes em } \\
\text { dois ou mais grupos } \\
\text { comparando-os }\end{array}$ \\
\hline $\begin{array}{l}\text { Bardella e } \\
\text { cols. }\end{array}$ & 2001 & $\begin{array}{l}\text { Construção de tabelas padronizadas } \\
\text { Relação entre traços de personalidade e } \\
\text { gênero, idade e/ ou nivel educacional }\end{array}$ & 211 & PMK & $\begin{array}{l}\text { Divisão dos participantes em dois on } \\
\text { mais grupos comparando-os }\end{array}$ \\
\hline Costa & 2001 & $\begin{array}{l}\text { Construção de tabelas padronizadas } \\
\text { Relação entre traços de personalidade e } \\
\text { gênero, idade e/ ou nivel educacional }\end{array}$ & 227 & PMK & $\begin{array}{l}\text { Divisão dos participantes em } \\
\text { dois ou mais grupos } \\
\text { comparando-os }\end{array}$ \\
\hline $\begin{array}{l}\text { Frasson e } \\
\text { Souza }\end{array}$ & 2002 & $\begin{array}{l}\text { Relação entre acidentes, infrações e } \\
\text { variáveis de personalidade. }\end{array}$ & 10 & Rorschach & $\begin{array}{l}\text { Um grupo de sujeitos e verificação do } \\
\text { resultado no(s) instrumento(s) }\end{array}$ \\
\hline $\begin{array}{l}\text { Esteves e } \\
\text { cols. }\end{array}$ & 2004 & $\begin{array}{l}\text { Comparação de tabelas padronizadas entre } \\
\text { diferentes estados }\end{array}$ & 356 & PMK & $\begin{array}{l}\text { Comparação entre amostras de } \\
\text { diferentes contextos brasileiros }\end{array}$ \\
\hline $\begin{array}{l}\text { Lamounier } \\
\text { e Rueda }\end{array}$ & $2005 \mathrm{a}$ & Estudos sobre validade e padronização & 110 & PMK & $\begin{array}{l}\text { Divisão dos participantes em } \\
\text { dois ou mais grupos } \\
\text { comparando-os }\end{array}$ \\
\hline $\begin{array}{l}\text { Ávila e } \\
\text { Benczik }\end{array}$ & 2005 & $\begin{array}{l}\text { Relação entre acidentes, infrações e } \\
\text { variáveis de personalidade }\end{array}$ & 1 & PMK & Estudo de caso \\
\hline Ral e cols. & 2005 & $\begin{array}{l}\text { Construção de tabelas padronizadas } \\
\text { Estudos sobre validade e padronização }\end{array}$ & 505 & PMK-RD & Estudo transcultural \\
\hline Ral e cols. & 2006 & Estudos sobre validade e padronização & 317 & PMK-RD & Estudo transcultural \\
\hline $\begin{array}{l}\text { Lamounier e } \\
\text { Rueda }\end{array}$ & 2006 & $\begin{array}{l}\text { Relação entre traços de personalidade e gênero, } \\
\text { idade e/ ou nivel educacional }\end{array}$ & 126 & $P M K$ & $\begin{array}{l}\text { Divisão dos participantes em dois ou } \\
\text { mais grupos comparando-os }\end{array}$ \\
\hline $\begin{array}{l}\text { Lamounier } \\
\text { e Villemor- } \\
\text { Amaral }\end{array}$ & 2006 & Estudos sobre validade e padronização & 42 & Rorschach & $\begin{array}{l}\text { Divisão dos participantes em } \\
\text { dois ou mais grupos } \\
\text { comparando-os }\end{array}$ \\
\hline
\end{tabular}

Com relação aos participantes nas pesquisas, o maior número foi de 4.935 (Vieira e cols., 1956), seguido de 505 (Ral e cols., 2005), 356 (Esteves e cols., 2004) e 317 (Ral e cols., 2006). Nos outros estudos, as amostras foram compostas com número inferior a 300 participantes. Evidencia-se, desse modo, a ausência de representatividade da população brasileira e de condutores, principalmente diante da apresentação de tabelas padronizadas (Bardella e cols., 2001; Costa, 2001; Ral e cols., 2005) e suas comparações com tabelas em diferentes estados (Boccalandro e cols., 1971; Esteves e cols., 2004). Considera-se que as propostas de uso de normas para uma única cidade/estado podem não ser consideradas exeqüíveis, pela ausência de parâmetros demográficos, não garantindo representatividade da população pesquisada. Além disso, o fato de serem observadas diferenças entre os resultados por sexo ou em tabelas para motoristas não bastam para assinalar a validade do processo avaliativo, pode demonstrar somente o que o instrumento está medindo, mas não 
demonstra que tais características são realmente necessárias na avaliação de candidatos a motoristas. Outro aspecto importante, é que nove, das quinze pesquisas, estudaram candidatos a habilitação e universitários (Alves e cols., 1998; Bardella e cols., 2001; Costa, 2001; Esteves e cols., 2004; Lamounier \& Rueda, 2005a, 2006; Ral e cols., 2005, 2006; Vieira e cols., 1956), sendo em menor número as que estudaram motoristas já habilitados propriamente (Ávila \& Benczik, 2005; Boccalandro e cols., 1971; Frasson \& Souza, 2002; Grisci, 1991; Hashimoto \& Ribeiro, 1991; Lamounier \& VillemorAmaral, 2006; Vieira e cols., 1956). Considera-se que aquelas amostras não representam a população-alvo (motoristas) e os resultados obtidos podem ser considerados meramente ilustrativos, impossíveis de generalização, não obstante a apresentação de tabelas normativas induza sua aplicação para além da amostra de referência. Outro aspecto a ser ressaltado é que os resultados dos estudos apresentam alguns indícios de condições psicopatológicas e de comportamentos exacerbados observados nos testes; todavia, as condições metodológicas utilizadas e a ausência de parâmetros amostrais não permitem afirmar efetivamente que esses sinais possam ser definidos como causa/conseqüência dos acidentes.

Dos instrumentos psicológicos citados, onze estudos utilizaram o PMK, dois deles com uma versão experimental digitalizada, o PMK-RD; duas investigações empregaram o Bender projetivo e dois trabalhos utilizaram o Rorschach. Chama atenção nesses dados a ocorrência de publicações apenas com instrumentos projetivos para o estudo da personalidade, não obstante se saiba que instrumentos psicométricos também são largamente utilizados no Brasil (Alchieri \& Stroeher, 2003). O instrumento de maior uso nas pesquisas foi o PMK, confirmando sua ampla utilização, mas sem a existência ou concordância de um perfil a avaliar (Alchieri $\&$ Stroeher, 2002). Destaca-se, ainda, o advento de uma forma digitalizada do teste, o que tem possibilitado publicações relacionando amostras brasileiras e espanholas, em que pese estar ainda em forma experimental, carecendo de novos estudos que possibilitem generalizações confiáveis. Os estudos sobre o Bender projetivo tentaram elaborar alguns indicadores e critérios, bem como adaptar seu uso para a avaliação de características de personalidade de condutores (Grisci, 1991; Hashimoto \& Ribeiro, 1991), todavia seus resultados não apresentam amparo empírico suficiente para sustentar suas conclusões, sendo a sugestão de indicação de uso respaldada por elementos incompletos e metodologicamente inadequados. Em relação ao Rorschach, é oportuno evidenciar que, embora apenas duas pesquisas tenham sido encontradas, sabe-se que sua utilização na seleção de motoristas ocorre desde a década de 1930, sendo muito usado em diversos estados (Alchieri \& Stroeher, 2003; Mange, 1956). Para o psicólogo menos experiente na área, pode parecer que o fato de haver alguma pesquisa sobre esses instrumentos (PMK, Bender projetivo e Rorschach) justifique o seu uso e, conseqüentemente, a avaliação da personalidade no trânsito; no entanto, é o perfil que recomenda o instrumento a adotar e, sobre isso, ressalta-se que nem mesmo a Resolução n 80/1998 do Conselho Nacional de Trânsito estabelece qualquer indicação de característica de personalidade que possa habilitar ou não um candidato (Alchieri \& Stroeher, 2003).

Quanto aos delineamentos adotados nas pesquisas, oito trabalhos utilizaram a comparação entre grupos (Alves e cols., 1998; Bardella e cols., 2001; Costa, 2001; Grisci, 1991; Lamounier \& Rueda, 2005a, 2006; Lamounier \& Villemor-Amaral, 2006; Vieira e cols., 1956); dois trabalhos utilizaram apenas um grupo de sujeitos, objetivando a aplicação e análise de resultados obtidos mediante o(s) instrumento(s) (Frasson \& Souza, 2002; Hashimoto \& Ribeiro, 1991); dois trabalhos realizaram análise comparativa em estudo transcultural (Ral e cols., 2005, 2006); outros dois realizaram análises comparativas entre amostras de diferentes contextos brasileiros (Boccalandro e cols., 1971; Esteves e cols., 2004) e um trabalho realizou estudo de caso (Ávila \& Benczik, 2005). A comparação foi o delineamento mais utilizado nos estudos. Outro aspecto a ser destacado é que apenas um estudo de caso foi identificado, não obstante o incremento desta modalidade de investigação com bons motoristas possa trazer contribuições importantes, não só em relação à gênese do comportamento seguro, mas também sobre o comportamento infrator. Não foram observados estudos com dados nacionais em delineamentos longitudinais e de follow-up que acompanham, por um período de tempo, os (futuros) motoristas, imprescindível para auxiliar na validade do processo psicológico.

\section{Conclusão}

Milhões de brasileiros são avaliados psicologicamente para conduzir veículos anualmente, porém, tomando-se a literatura apresentada e analisada na revisão, verifica-se que as pesquisas sobre personalidade de motoristas são escassas e bastante restritas quanto a generalizações, não apresentando resultados conclusivos que justifiquem a necessidade de avaliação do construto para conduzir veículos. Diante disso, a discussão quanto às características de personalidade dos candidatos a condutores e condutores revela-se estéril diante do quadro de pesquisas anteriormente conduzidas, uma vez 
que a validade na indicação de um teste está nos estudos aprimorados e controlados metodologicamente, tendo como foco as condições comportamentais na atividade de dirigir e não somente na apresentação de resultados em tabelas normativas. Os esforços de alguns poucos estudiosos definiram a pesquisa sobre avaliação psicológica de motoristas no Brasil, denotando que muito ainda necessita ser realizado. Reverter essa situação passa pelo investimento na elaboração de perfil (ou perfis) de motorista(s) quanto aos aspectos da personalidade, por estudos sobre a validade e fidedignidade dos testes de personalidade atualmente utilizados, pelo desenvolvimento de melhores instrumentos de avaliação ou, mesmo, pelo delineamento de uma alternativa metodológica para a avaliação psicológica. Outro aspecto decisivo para reverter este quadro, é o incremento de ações integradas ou parcerias entre a universidade e as instituições responsáveis pelo trânsito e transporte rodoviário, como órgãos estaduais e municipais de trânsito, empresas de transporte coletivo municipal e interestadual, empresas de seguro e cooperativas de motociclistas, táxis ou de transportes alternativos, possibilitando representatividade amostral das populações investigadas. Mediante esta sistematização, espera-se contribuir na elaboração de novos procedimentos de investigação e no desenvolvimento da psicologia do trânsito, possibilitando o fomento de esforços mais efetivos e substanciais sobre o construto personalidade.

\section{Referências}

Alchieri, J. C. \& Scheffel, M. (2000). Indicadores da produção científica brasileira em avaliação psicológica: resultados da elaboração de uma base de dados dos artigos publicados em periódicos brasileiros de 1930 a 1999. Anais do V Encontro Mineiro de Avaliação Psicológica e VIII Conferência Internacional de Avaliação Psicológica, 195197.

Alchieri, J. C. \& Stroeher, F. (2002). Avaliação psicológica no trânsito: o estado da arte no Brasil sessenta anos depois. Em R. M. Cruz, J. C. Alchieri \& J. Sardá (Orgs.). Avaliação e medidas psicológicas (pp. 234-345). São Paulo: Casa do Psicólogo.

Alchieri, J. C. \& Stroeher, F. (2003). Características do processo de avaliação psicológica para condutores de veículos: um estudo sobre a tomada de decisão dos psicólogos na utilização de testes psicológicos no Brasil. Revista Iberoamericana de Diagnóstico y Evaluación Psicológica, 15(1), 107-119.

Alves, I. C. B. (1999). O exame psicotécnico de motoristas. Anais do VIII Congresso Nacional de Avaliação Psicológica, Porto Alegre, 216-224.

Psico-USF, v. 12, n. 2, p. 189-196, jul./ dez. 2007
Alves, I. C. B. e cols. (1998). O psicodiagnóstico miocinético em motoristas de três níveis de escolaridade. Boletim de Psicologia, 48(109), 1-24.

Arsênio, J. (1999). Dilemas da avaliação psicológica. Anais do VIII Congresso de Avaliação Psicológica, Porto Alegre, 225228.

Ávila, F. A. \& Benczik, E. B. P. (2005). Transtorno de Déficit de Atenção-Hiperatividade - TDAH: um estudo do exame psicológico de um motorista infrator. Revista ABR AMET, 22(45), 32-39.

Bardella, J. G., Alves, I. C. B., Esteves, C., Guedes, M. B. B. \& Almeida, Y. D. B. (2001). Psicodiagnóstico Miocinético - PMK: normas para motoristas da cidade do Recife (PE). PSIC: Revista de Psicologia da Vetor Editora, 2(2/3), 106-137.

Boccalandro, E. R., Tolentino, C. M. S., Nogueira, D. F., Maia, E. M. \& Lima, M. J. (1971). O PMK em motoristas profissionais. Boletim de Psicologia 23(62), 7-15.

Costa, F. R. (2001). PMK: estudos de normatização de uma nova amostra para candidatos à Carteira Nacional de Habilitação. PSIC: Revista de Psicologia da Vetor Editora, 2(1), 4-44.

Duarte, T. O. (1999). Validade preditiva dos testes Atenção Concentrada Toulouse-Pierón, Bender e IFP na avaliação do comportamento de motoristas. Dissertação de Mestrado. Rio Grande do Sul: Universidade Federal do Rio Grande do Sul.

Esteves, C., Bardella, M. B., Almeida, Y. D. B., Bardella, J. G. \& Alves, I. C. B. (2004). Comparação dos resultados do psicodiagnóstico miocinético - PMK de Belo Horizonte com os de Recife e São Paulo. PSIC: Revista de Psicologia da Vetor Editora, 5(2), 36-47.

Frasson, L. M. M. \& Souza, M. A. (2002). Estudo qualitativo da personalidade do motorista infrator através do Rorschach e EDAO. Boletim de Psicologia, 52(117), 141-157.

Gouveia, V. V., Silva, D. V., Silva, M. P. V., Andrade, M. V. C. L., Silva Filho, S. B. \& Costa, D. M. F. (2002). Atitudes frente à avaliação psicológica para condutores: perspectivas de técnicos, estudantes de psicologia e usuários. Psicologia: Ciência e Profissão, 22(2), 50-59.

Grisci, C. L. I. (1991). Relação entre acidentes de trânsito e as variáveis de agressividade, atuação e culpa. PSICO, 21(1), 103-117.

Groeger, J. A. (2003). Testes psicológicos podem predizer habilidade para dirigir? Em R. Risser (Org.). Estudos sobre a avaliação psicológica de motoristas (pp. 7995). São Paulo: Casa do Psicólogo. 
Hashimoto, F. \& Ribeiro, C. (1991). O Bender projetivo na seleção de motoristas. Boletim de Psicologia, 41(94/95), 15-26.

Lamounier, R. \& Rueda, F. J. M. (2005a). Avaliação psicológica com o PMK no contexto do trânsito. Psicologia: Pesquisa \& Trânsito, 1(1), 25-32.

Lamounier, R. \& Rueda, F. J. M. (2005b). Avaliação psicológica no trânsito: perspectiva dos motoristas. PSIC: Revista de Psicologia da Vetor Editora, 6(1), 35-42.

Lamounier, R. \& Rueda, F. J. M. (2006). O psicodiagnóstico miocinético em motoristas: diferenças por gênero, escolaridade e idade. Avaliação Psicológica, 5(2), 179-187.

Lamounier, R. \& Villemor-Amaral, A. E. (2006). Evidencias de validez para el Rorschach en el contexto de la psicología de transito. Revista Interamericana de Psicología, 40(2), 167-176.

Mange, R. (1956). Evolução da psicotécnica em São Paulo. Arquivos Brasileiros de Psicotécnica, 8(1), 5-7.

Méa, C. P. D. \& Ilha, V. D. (2003). Percepção de psicólogos do trânsito sobre a avaliação de condutores. Em M. H. Hoffmann, R. M. Cruz \& J. C. Alchieri (Orgs.). Comportamento bumano no trânsito (pp. 263-288). São Paulo: Casa do Psicólogo.

Mira, A. M. G. (1984a). Resultados das pesquisas realizadas com o PMK em motoristas pluriacidentados e não ou pauciacidentados. Psicologia \& Trânsito, 1(2), 23-32.

Mira, A. M. G. (1984b). Critérios para qualificar um candidato a motorista psicologicamente inapto pelo teste PMK. Psicologia \&o Trânsito, 1(2), 53-56.

Quintela, G. (1977). A validade do psicotécnico na seleção de motoristas, quando cientificamente aplicado e interpretado o psicodiagnóstico miocinético de Mira y López. Arquivos Brasileiros de Psicologia Aplicada, 29(3), 137-142.

Ral, J. M. T., Sanzano, A. V. \& Martinez, R. M. (2005).
Normalização dos lineogramas do PMK para Barcelona e sua comparação com Recife. PSIC: Revista de Psicologia da Vetor Editora, 5(2), 1-15.

Ral, J. M. T., Viadé, A. \& Muiños, R. (2006). Comparação dos lineogramas do PMK-RD com os lineogramas do PMK. Psicologia: Pesquisa \& Trânsito, 2(1), 1-8.

Rozestraten, R. J. A. (1982). A opinião de psicólogos brasileiros sobre o exame psicológico para motorista no Brasil. Anais da XII Reunião Anual de Psicologia, Ribeirão Preto, 105-117.

Rozestraten, R. J. A. (1985). O exame psicológico para motorista em alguns países fora do Brasil. Psicologia \& Trânsito, 2(2), 67-74.

Rozestraten, R. J. A. (1990). O uso e a validade dos testes aplicados aos motoristas - dados de uma pesquisa. Anais da $20^{a}$ Reunião Anual de Psicologia, Ribeirão Preto, 471476.

Sbardelini, E. T. B. (1990). O uso e a validade dos testes psicológicos na seleção de motoristas - formação do psicólogo para atuar em psicotécnico. Anais da 20 Reunião Anual de Psicologia, Ribeirão Preto, 467-470.

Trench, G. (1956). Resultados da seleção e formação profissional na CMTC de São Paulo. Arquivos Brasileiros de Psicotécnica, 8(1), 97-105.

Vieira, M. V. M., Amorim, J. A. \& Carvalho, A. V. (1956). $\mathrm{O}$ psicodiagnóstico miocinético na seleção de motoristas. Arquivos Brasileiros de Psicotécnica, 8(1), 53-65.

Wechsler, S. M. (1999). Aspectos éticos e deontológicos na avaliação psicológica: implicações para o psicotécnico. Anais do VIII Congresso Nacional de Avaliação Psicológica, Porto Alegre, 229-233.

Recebido em maio de 2007 Reformulado em agosto de 2007 Aprovado em setembro de 2007

Sobre os autores:

Fábio Henrique Vieira de Cristo e Silva é psicólogo, mestrando na UFRN, coordenador da Comissão de Psicologia do Trânsito do CRP-17 e colunista dos sites Portal do Condutor e Digizap sobre comportamento no trânsito.

João Carlos Alchieri é psicólogo, doutor em Psicologia e professor do Programa de Pós-Graduação em Psicologia da UFRN. 